\title{
Exercise induced hemoconcentration following spleen contraction in subjects with COPD
}

\author{
Erika Schagatay ${ }^{1,2}$, Anna Hubinette ${ }^{1}$, Angelica Lodin-Sundström ${ }^{1,2}$, Harald Engan ${ }^{1,2,3^{*}}$ and Nikolai Stenfors ${ }^{4}$
}

\begin{abstract}
Background: The blood-boosting spleen contraction represents a potential protective response to hypoxia by raising the blood gas storage capacity. Human spleen contraction has been observed during exercise, apnea and simulated altitude resulting in ejection of stored red blood cells into circulation. High-altitude exposure has been shown to increase spleen contraction suggesting that long-term hypoxia may improve the response in humans. Subjects with COPD are often exposed to hypoxia, which limits their physical performance. However, it is not known if spleen contraction occurs in subjects with COPD. Our aim was to reveal whether subjects with COPD recruit the spleen erythrocyte reserve during mild exercise.
\end{abstract}

Methods: $\mathrm{SpO}_{2}$, spleen volume and $\mathrm{Hb}$ were measured before and after 6 min walking test (6MWT) in 24 subjects with COPD. Results were analyzed for all subjects pooled and for subject groups with resting $\mathrm{SpO}_{2}$ above and below $90 \%$ separated and expressed as mean.

Results: $6 \mathrm{MWT}$ reduced $\mathrm{SpO}_{2}$ from 91 to $83 \%$ and spleen volume from 254 to $181 \mathrm{~mL}$, while $\mathrm{Hb}$ increased from 150 to $154 \mathrm{~g} / \mathrm{L}$ ( $p=0.001$ for all). Compared to subjects with $\mathrm{SpO}_{2}>90 \%$, the group with $\mathrm{SpO}_{2}<90 \%$ displayed the largest resting spleen volume (339 vs $202 \mathrm{~mL} ; p=0.001$ ) and the most pronounced spleen volume reduction (139 vs $40 \mathrm{~mL} ; p=0.007$ ).

Conclusion: Exercise with hypoxia evokes spleen contraction in subjects with COPD and may represent a protective response during periods of hypoxia. The larger spleen volume and more pronounced contraction in the most hypoxic subjects may suggest long-term adaptation to hypoxia.

Keywords: Hypoxia, Erythrocyte reservoir, Hemoglobin, Physiological stress, Protective

\section{Background}

The mammalian spleen functions as an erythrocyte reservoir recruited via spleen contraction during physiological stress [1]. In e.g. diving seals, spleen contraction may prolong aerobic dive duration $[2,3]$. In healthy human subjects, spleen contraction and circulating hemoglobin $(\mathrm{Hb})$ increase have also been observed during apnea [4-7]. Interestingly, apnea in splenectomized subjects was found not to induce a $\mathrm{Hb}$ increase [5], and neonates with asplenia showed impaired transient elevation of blood $\mathrm{Hb}$ in

\footnotetext{
* Correspondence: Harald.Engan@miun.se

'Department of Health Sciences, Mid Sweden University, Östersund, Sweden ${ }^{2}$ Swedish Winter Sports Research Centre, Mid Sweden University, Östersund, Sweden

Full list of author information is available at the end of the article
}

response to acute hypoxia [8]. Bakovic and associates concluded that the contraction is an active process, as no differences in blood flow in the splenic artery or vein occurred [9], and total plasma protein levels remained unchanged across apnea series ruling out extravasation of plasma [5].

Spleen-contraction-induced increases in $\mathrm{Hb}$ concentration may have beneficial effects during hypoxia, e.g. prolonging apnea duration [4]. Splenic contribution of $\mathrm{Hb}$ to the circulating blood volume has also been observed in healthy subjects during exercise [10-12] and more recently during altitude simulation by normobaric hypoxia [13, 14]. This suggests that spleen contraction may be an emergency response triggered in a number of circumstances involving hypoxia. Both hypoxia and hypercapnia will enhance spleen contraction during apnea, 
with the net effect being greatest with both stimuli present [13-15]. Injection of catecholamines has also been shown to induce spleen contraction, suggesting that it is also a general stress response $[16,17]$.

In competitive apnea divers, it has been noted that the best divers have the largest spleens involving volumes of up to $500 \mathrm{~mL}$ and ejection of $250 \mathrm{~mL}$ of blood [18] with a hematocrit of about twice normal arterial hematocrit into circulation during apnea [19]. Large spleen volume and the ability to contract the spleen in apneic divers frequently exposed to hypoxia may reflect either inherent predisposition, or possibly a training effect. Both the elevated oxygen $\left(\mathrm{O}_{2}\right)$ storage and carbon dioxide $\left(\mathrm{CO}_{2}\right)$ buffering capacity associated with the enhanced hematocrit would be beneficial for prolonging apneic duration by delaying asphyxia.

The ability to withstand acute and chronic hypoxemia is important in several medical conditions, such as respiratory disorders. Subjects with chronic obstructive pulmonary disease (COPD) may have acute, intermittent and/or chronic hypoxemia [20], which limits their physical performance [21]. In subjects with COPD, a dynamic spleen reservoir increasing circulating erythrocyte volume and blood $\mathrm{O}_{2}$ storage capacity would likely be beneficial e.g. during transient periods of physiological strain and also to reduce blood viscosity by filtration of erythrocytes back into the spleen after its re-expansion during periods of rest. It is unknown, however, whether spleen contraction occurs in subjects with COPD, to what degree and under what circumstances. Our aim was to reveal whether subjects with COPD recruit the spleen erythrocyte reserve during mild exercise, and whether spleen volume varies with the level of hypoxia during exercise.

\section{Methods}

\section{Subjects}

The study was carried out at the Department of Respiratory Medicine at Östersund Hospital, Sweden. The ambition was to include a convenient sample representing subjects with moderate to very severe COPD with patients acting as their own controls, and with a possibility to compare responses between subjects with different levels of $\mathrm{O}_{2}$ saturation. Twenty-four subjects, 9 men and 15 women with moderate to very severe COPD [20], were recruited in connection with an elective outpatient visit. A description of study subjects is presented in Table 1. All subjects had taken their bronchodilators (short-acting $\beta$-agonist, long-acting $\beta$-agonist, ipatropium, and/or tiotropium) at least one hour prior to the start of the study protocol. Eight subjects had taken their morning beta-blockers prior to the protocol. Four subjects were on long-term $\mathrm{O}_{2}$ therapy 16-20 h/day and arrived to the outpatient department without their $\mathrm{O}_{2}$ therapy, and did not receive $\mathrm{O}_{2}$ therapy during the study measurements. Twenty-one subjects were ex-smokers, two current smokers and one subject had never smoked regularly.

Common co-morbidities were hypertension (seven subjects), coronary heart disease (six), and diabetes (four). One subject had alpha-one-antitrypsin deficiency and one had known pulmonary hypertension. Written informed consent was obtained, and the study had been approved by the regional ethics committee at Umeå University, Sweden.

\section{Protocol}

The work test protocol consisted of $10 \mathrm{~min}$ of sitting rest, followed by the subject standing up for two min and a six min walking test (6MWT) [22] and an additional $10 \mathrm{~min}$ of sitting rest. The 6MWT is commonly used in clinical practice and clinical trials of lung disease. The 6MWT was done by walking back and forth on a marked $30 \mathrm{~m}$ track at a speed chosen by the subject, after the instruction to try to cover as much distance as possible in $6 \mathrm{~min}$. The subject was accompanied by an assistant walking at arm's length for safety.

\section{Measurements}

On the arrival at the Respiratory Department spirometry was done to measure forced $\mathrm{FEV}_{1}$ (forced expiratory volume first second) and FVC (forced expiratory volume). Heart rate (HR) and peripheral oxygen saturation $\left(\mathrm{SpO}_{2}\right)$ were measured continuously across the protocol, using a portable pulse-oxymeter (Wristox Nonin 9500 Onyx, Nonin Medical Inc., MN, USA). During the resting periods before and directly after the 6MWT, spleen maximal length (L) thickness (T) and width (W) were measured using ultrasonic imaging (Mindray DP-6600, Shenzhen Mindray Bio-Medical Electronics Co., Ltd., Shenzhen, China) every minute for volume calculations using the formula $\mathrm{L} \pi\left(\mathrm{WT}-\mathrm{T}^{2}\right) / 3$ [7] . The formula is based on the average shape of the spleen, and describes the difference between two ellipsoids divided by two. Capillary blood samples were drawn from the finger after the initial $10 \mathrm{~min}$. rest, directly after 6MWT and $10 \mathrm{~min}$ post walking for $\mathrm{Hb}$, which were directly analyzed in triplicate (Hemocue Hb 201+, Hemocue AB, Ängelholm, Sweden) and mean values used.

\section{Analysis}

As no gender effects were found, genders were pooled. Analysis of all variables was done for the whole group of 24 subjects, and also for subjects separated into "nonhypoxic" (resting $\mathrm{SpO} 2>90 \% ; n=15 ; 7$ males) and "hypoxic" (resting $\mathrm{SpO} 2<90 \% ; n=9 ; 2$ males) COPD groups. Normality of distribution was checked with 
Shapiro-Wilks test and normal probability plots. As some variables were not normally distributed, nonparametric tests were used in all analyses. Two-sided paired Wilcoxon test with Bonferroni correction for multiple corrections was used to compare the baseline versus post $6 \mathrm{MWT}$ values. The individual values of spleen volume, $\mathrm{Hb}$ and $\mathrm{SpO}_{2}$ were analyzed for correlation both pre and post 6MWT with Spearman's rank correlation coefficient. Pre values for spleen volume was determined from the last minute before the apnea onset. Mann-Whitney U test was used for comparisons between groups. Significance was accepted at $p<0.05$.

\section{Results}

Baseline $\mathrm{SpO}_{2}$ correlated to spleen volume $(r=-0.55$, $p=0.005)$ and $\mathrm{SpO}_{2}$ post exercise to spleen volume reduction $(r=-0.62, p=0.001)$.

Spleen volume was reduced along with $\mathrm{SpO}_{2}$ by the $6 \mathrm{MWT}(p<0.001$; Fig. 1$)$ and changes were reversed within $10 \mathrm{~min}$ after the test. $\mathrm{Hb}$ was $150 \mathrm{~g} / \mathrm{L}$ at baseline and had increased by $3.9 \mathrm{~g} / \mathrm{L}$ after walking $(p=0.001)$ and after $10 \mathrm{~min}$ recovery $\mathrm{Hb}$ remained elevated by $1.4 \mathrm{~g} / \mathrm{L}(p=0.002$; Fig. 2). Baseline spleen volume and $\mathrm{Hb}$ were correlated to baseline $\mathrm{SpO}_{2}(r=-0.55, p=$ 0.005 and $r=-0.50, p=0.013$, respectively). Spleen volume reduction by the $6 \mathrm{MWT}$ was correlated to spleen volume at baseline $(r=0.69 ; p=0.001)$ and $\mathrm{SpO}_{2}$ post $6 \mathrm{MWT}(r=-0.62, p=0.001)$, but not to the reduction in $\mathrm{SpO}_{2}$ across the test $(r=0.30$; NS) or to the $\mathrm{Hb}$ increase $(r=-0.19 ; \mathrm{NS})$. The degree of desaturation $\left(\Delta \mathrm{SpO}_{2}\right)$ during 6MWD was not correlated with the changes of spleen volume $(r=0.338$; NS) or $\mathrm{Hb}(r=-0.32$; NS) from baseline.

During the walking test, mean (SD) heart rate increased from 81 (16) to 105 (19) beats per minute $(p=0.001)$ and after $10 \mathrm{~min}$ recovery it was still slightly elevated at 83 (16) beats per minute $(p=0.009)$. The subjects covered 297 (95) meters in the 6MWT.

When "hypoxic" $(n=9)$ and "non-hypoxic" $(n=15)$ groups were compared, the "hypoxic" group displayed

Table 1 Description of the 24 subjects

\begin{tabular}{|c|c|c|c|c|}
\hline & All $(N=24)$ & Hypoxic $(N=9)$ & Non-hypoxic $(N=15)$ & $p$-value \\
\hline Male (N) & 9 & 2 & 7 & \\
\hline Age (years) & $71(8.8)$ & $72(8.2)$ & $71(9.0)$ & 0.758 \\
\hline Height (cm) & $166(8.6)$ & $161(8.7)$ & $168(7.4)$ & 0.034 \\
\hline Weight (kg) & $69(14.6)$ & $75(14.3)$ & $66(14.1)$ & 0.145 \\
\hline BMI $\left(\mathrm{kg} / \mathrm{m}^{2}\right)$ & $25(5.5)$ & $29(5.7)$ & $23(4.1)$ & 0.008 \\
\hline $\mathrm{FEV}_{1}$ (Liters) & $0.85(0.32)$ & $0.94(0.31)$ & $0.8(0.32)$ & 0.308 \\
\hline $\mathrm{FEV}_{1}$ (\% of predicted) & $33(11.7)$ & $39(12.2)$ & $30(10.3))$ & 0.053 \\
\hline FVC (Liters) & $1.7(0.5)$ & $1.9(0.6)$ & $1.7(0.4)$ & 0.481 \\
\hline FVC (\% of predicted) & $52(14.8)$ & $60(17.6)$ & $47(10.4)$ & 0.026 \\
\hline $\mathrm{FEV}_{1} / \mathrm{FVC}$ & $0.49(0.1)$ & $0.52(0.1)$ & $0.47(0.1)$ & 0.290 \\
\hline $\mathrm{PaO}_{2}(\mathrm{kPa})$ & $8.8(1.5)$ & $7.4(0.8)$ & $9.5(1.2)$ & 0.001 \\
\hline $\mathrm{PaCO}_{2}(\mathrm{kPa})$ & $5.4(0.6)$ & $5.6(0.5)$ & $5.2(0.6)$ & 0.034 \\
\hline 6-min walking test (meters) & $297(95)$ & $267(87)$ & $314(98)$ & 0.237 \\
\hline LTOT (N) & 4 & 3 & 1 & \\
\hline LAMA (N) & 14 & 6 & 8 & \\
\hline ICS (N) & 19 & 7 & 12 & \\
\hline SAMA (N) & 6 & 4 & 2 & \\
\hline SABA (N) & 9 & 3 & 6 & \\
\hline Hypertension (N) & 7 & 4 & 3 & \\
\hline Coronary heart disease $(\mathrm{N})$ & 6 & 3 & 3 & \\
\hline Diabetes (N) & 4 & 2 & 2 & \\
\hline Claudicatio intermittens (N) & 2 & 1 & 1 & \\
\hline Current smoker (N) & 2 & 2 & & \\
\hline Former smoker (N) & 22 & 7 & 15 & \\
\hline
\end{tabular}

Table text: Data presented as mean (SD). Lung function volumes are post-bronchodilation values. BMI body mass index, FEV forced expiratory volume, FVC forced vital capacity, $\mathrm{PaO}_{2}$ partial pressure of arterial oxygen, $\mathrm{PaO}_{2}$ partial pressure of arterial carbon dioxide, LTOT long-term oxygen therapy, LAMA long-acting muscarinic antagonist, ICS inhaled corticosteroid, LABA long-acting beta2-agonist, SAMA short-acting muscarinic antagonist, SABA short-acting beta2-agonist, $N$ number of subjects 


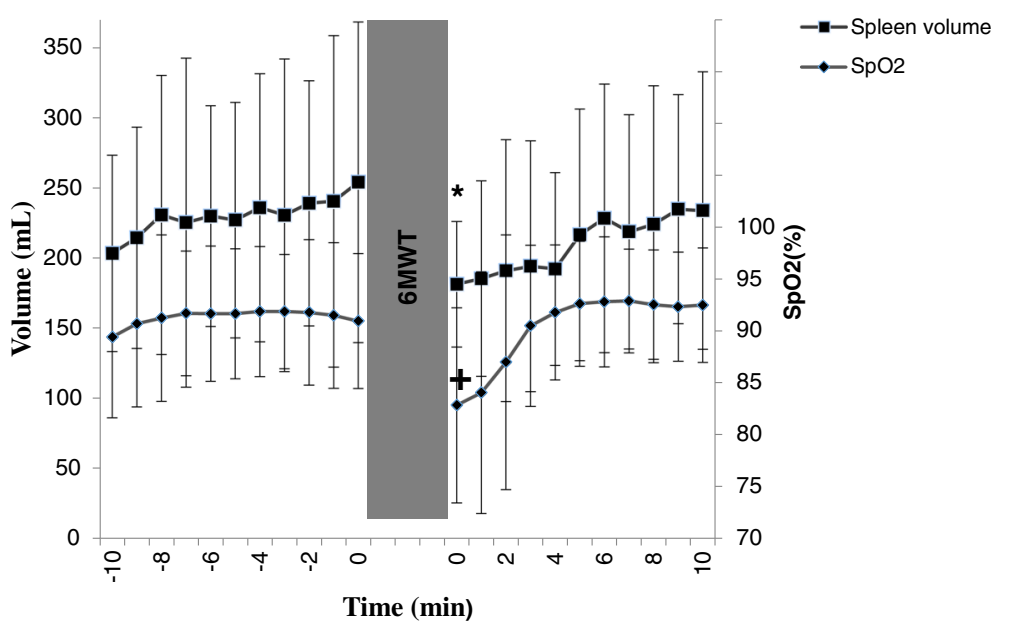

Fig. 1 Mean $\mathrm{SpO}_{2}$ and spleen volume (IQR) before and after the 6 min walking test (6MWT) in 24 subjects with COPD. $P<0.001$ is indicated with * for $\mathrm{SpO}_{2}$ and $P<0.01$ with + for spleen volume compared with pre-walking baseline values

the largest mean resting spleen volume, $339 \mathrm{~mL}$, versus $202 \mathrm{~mL}$ in the "non-hypoxic" group ( $p=0.001$; Fig. 3). The "hypoxic" group also had higher mean Hb concentration than the "non-hypoxic" group at $160 \mathrm{~g} / \mathrm{L}$ and $144 \mathrm{~g} / \mathrm{L}$, respectively $(p=0.003)$. They also responded to walking with a more pronounced spleen volume reduction, by $139 \mathrm{~mL}$ (36 \%), compared to subjects with "nonhypoxic" COPD, which displayed a mean reduction of $40 \mathrm{~mL}$ (19 \%; $p=0.007$; Fig. 3). The reduction in $\mathrm{SpO}_{2}$ was of the same magnitude; $9.7 \%$ in "hypoxic" and $9.4 \%$ in "non-hypoxic" subjects (NS). After walking, the "hypoxic" COPD group had a mean $\mathrm{Hb}$ increase of $4.5 \mathrm{~g} / \mathrm{L}$, compared to $3.5 \mathrm{~g} / \mathrm{L}$ for the "non-hypoxic" group (NS).
There was no difference in the distance covered in the 6MWT between "hypoxic" and "non-hypoxic" and COPD groups (267 m versus $314 \mathrm{~m}$; NS). HR responses were also similar in both groups (NS).

\section{Discussion}

The principal original findings of this study were that exercise evokes spleen contraction and $\mathrm{Hb}$ increase in subjects with COPD, and that the spleen response is enhanced in hypoxic subjects with COPD. The latter finding suggests that this spleen function may be essential for these individuals during hypoxic challenges similar to that found in healthy subjects during maximal exercise [23], high altitude simulation $[13,14]$ or apnea $[4,5,7]$. In

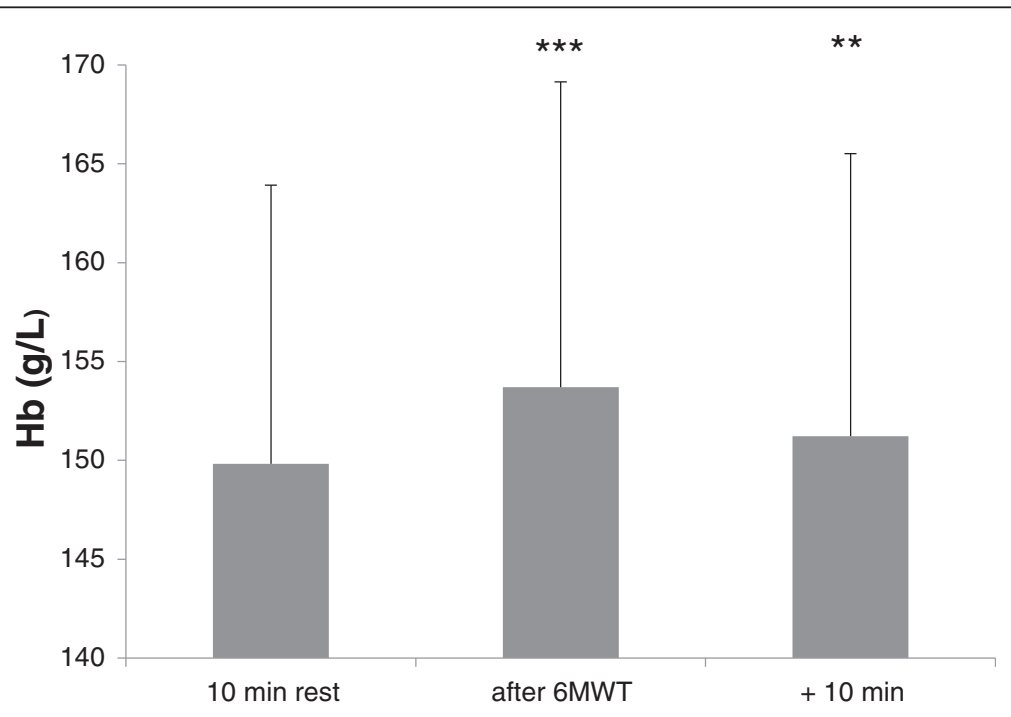

Fig. 2 Mean (IQR) hemoglobin concentration ( $\mathrm{Hb})$ after 10 min rest, after 6 min walking and after 10 min recovery in 24 subjects. $P<0.001$ is indicated with ${ }^{* *}$ and $P<0.01$ with ${ }^{* *}$ compared with values obtained after 10 min rest 


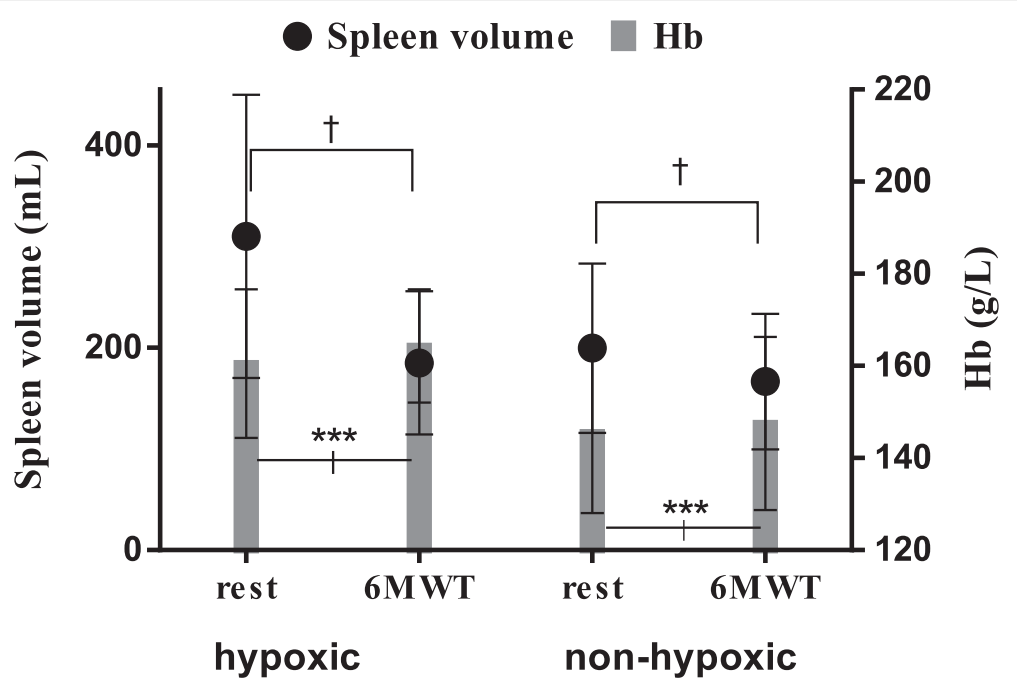

Fig. 3 Mean (IQR) hemoglobin concentration ( $(\mathrm{Hb})$ and spleen volume after 10 min rest and immediately after 6 min walking (6MWT) in hypoxic subjects (baseline $\mathrm{SpO}_{2}<90 \%$ ) and non-hypoxic subjects (baseline $\mathrm{SpO}_{2}>90 \%$ ). ${ }^{* * *}$ indicates $P<0.001$ for $\mathrm{Hb}$ values and $+P<0.01$ for spleen volume values

apneic diving, these responses have been shown to be protective against acute hypoxia $[5,18]$, and we suggest this may be the case also in COPD.

The correlation between spleen volume reduction and the hypoxic load of the disease is in line with observations in high altitude climbers, which were found to have enhanced spleen and $\mathrm{Hb}$ responses after a 2 month expedition to Mt Everest [24]. These results suggest that the spleen's ability to transfuse stored red blood cells into circulation may increase by training or long-term hypoxic exposure.

Subjects with $\mathrm{SpO}_{2}>90$ \% ("non-hypoxic" group) had spleen volumes in the same range as previously reported for the healthy population [25], while their spleen volume was about $30 \%$ larger in the subjects with $\mathrm{SpO}_{2}<$ $90 \%$ (hypoxic group); in the range of that observed in trained apnea divers [18]. This may suggest that baseline spleen volume could be increased by long-term exposure to chronic hypoxia. However, no increase in spleen volume was observed after the 2 month high altitude expedition [24] although it was previously observed that the best competition freedivers had larger spleens than less successful ones, which could suggest the spleen increases in size when frequently stimulated to contract [26]. In the "hypoxic" subjects with a larger spleen, baseline $\mathrm{Hb}$ was also higher than in the "non-hypoxic" group, possibly suggesting that the increased spleen volume may be a response to polycythemia. However, the high altitude climbers had not increased their baseline spleen volumes despite profound polycythemia as a result of the 2 month climb [24], thus any such changes would possibly occur on longer term exposure.

The $36 \%$ spleen contraction seen in the "hypoxic" group was of twice the magnitude compared to the $19 \%$ in the group with the "non-hypoxic" group. A $16 \%$ spleen volume reduction was previously reported in healthy subjects during normobaric hypoxia, but a $34 \%$ reduction was observed in the same subjects during maximal voluntary apnea at rest [14], despite a similar fall in $\mathrm{SpO}_{2}$ of approximately $10 \%$ in the two conditions. A similar fall in $\mathrm{SpO}_{2}$ was present in subjects with COPD, but a fall from a lower initial $\mathrm{SpO}_{2}$ level in the "hypoxic" group could possibly cause a stronger hypoxic stimulus causing the more pronounced contraction. Due to greater baseline spleen volume in the "hypoxic" group, the actual volume expelled differed more from baseline, with a mean $139 \mathrm{~mL}$ volume reduction compared to the "non-hypoxic" group, which displayed a mean volume reduction of $40 \mathrm{~mL}$. Thus in the "hypoxic" group, the extra red cell volume was likely 3 times greater, which was also reflected by a greater increase in $\mathrm{Hb}$.

The "hypoxic" group responded with a spleen volume reduction of $139 \mathrm{ml}$, which is in the range previously found in trained divers [18], and found to have a significant physiological effect $[5,18]$. Considering that the spleen contains blood with about twice the $\mathrm{Hb}$ as normal blood, the release of red cells would be equivalent to approximately $200 \mathrm{~mL}$ of blood, increasing the $\mathrm{O}_{2}$ carrying capacity by $4 \%$. This is also supported by the observed elevation of $\mathrm{Hb}$ by $4 \%$. Such an increase in $\mathrm{O}_{2}$ carrying capacity is likely to have a significant effect on $\mathrm{O}_{2}$ delivery to the tissues in subjects with chronic hypoxia due to lung disease - similar to as during high altitude exposure.

Hypoxia has been shown to be an important trigger of spleen contraction, although not the only factor involved $[14,27]$. The magnitude of spleen contraction in the present study was correlated to the degree of exerciseinduced and aggravated hypoxia. It is uncertain why the 
magnitude of spleen contraction was not correlated to the degree of desaturation $\left(\Delta \mathrm{SpO}_{2)}\right.$ during 6MWD. One reason could be that the COPD patients were slightly hypoxic at rest, possibly reducing the impact of chemoreception on spleen contraction during exercise. It should be mentioned that the larger degree of spleen contraction previously seen after maximal apnea compared to eupneic hypoxia may reflect an additional influence of hypercapnia $[14,15]$.

The mean $\mathrm{Hb}$ increase of $3.9 \mathrm{~g} / \mathrm{L}$ after exercise/ $6 \mathrm{MWT}$ in the present study was similar in magnitude compared to previous observations in healthy subjects during apnea [5, 7, 27], exercise [12] and eupneic hypoxia [13]. This response was more pronounced in COPD subjects with a larger spleen suggesting that spleen volume has an impact on the ability to expel erythrocytes. The Hb-response was also enhanced in subjects with pronounced $\mathrm{SpO}_{2}$ reduction after exercise, suggesting that splenic contraction with erythrocyte expulsion is closely related to the degree of hypoxia, in accordance with the study of Richardson et al.,2009 [27]. We suggest that spleen contraction with erythrocyte expulsion may attenuate hypoxia during exercise in subjects with COPD, similar to the effect found during apnea [5, 7]. An important question is whether the responses observed in the present study will be modified by repeated or prolonged exercise, or by long-term hypoxia.

The 6MWT represents a common exercise test in COPD. It reflects maximal exercise capacity, as subjects are asked to walk as far as possible during the $6 \mathrm{~min}$ walk, whilst they are able to regulate the intensity throughout the test. Whether an exercise-induced spleen response is observed at lower levels of intensity or shorter duration remains to be seen.

Spleen contraction may temporarily contribute to the high $\mathrm{Hb}$ associated with secondary polycytemia, a common condition in COPD [28], which may have harmful consequences related to hyperviscosity and raised pulmonary arterial pressure [29]. Spleen contraction may be involved in exacerbation of shear stress imposed by atherosclerotic plaques and plaque ruptures by increasing blood viscosity during physiological and psychological stress $[30,31]$, and clearly more research is needed to elucidated the spleen's role in regulation of stress related hemoconcentration.

The observation that COPD subjects with low baseline $\mathrm{SpO}_{2}$ had a larger spleen could possibly suggest that intermittent or chronic hypoxia may by a "training effect" lead to spleen enlargement, corresponding to observations of large spleens in the best apneic divers [18]. It could, however, also possibly be a consequence of venous pooling to the abdominal organs due to concurrent pulmonary hypertension and right ventricular heart failure, prevalent in many subjects with very severe COPD
[32]. A contributing effect of such underlying conditions, although not evident in the patient group studied, cannot be completely ruled out and should be investigated.

As subjects with COPD may have frequent episodes of hypoxemia and thus erythropoietic stress, an enlargement could possibly also be a consequence of extramedullary erythropoesis, or related to hyperplasia of the reticuloendothelial system due to increased destruction of defective erythrocytes [33]. An alternative, and in our view more likely, interpretation of the role of the spleen in this context is that it may protect subjects with COPD and secondary polycytemia against negative consequences related to hyperviscosity, via the splenic erythrocyte storage of excessive red blood cells from circulation during rest. Yet another tentative explanation could be that subjects with COPD with inherently larger spleens sustain the disease better and live longer, and thus are overrepresented in the severe stages of the disease. This observation deserves additional studies in larger materials over time to be fully understood. Since the elevation of $\mathrm{Hb}$ increases $\mathrm{O}_{2}$ carrying capacity while also involves an increase in blood viscosity which may lead to increased cardiovascular stress, further research will need to clarify whether the consequences are beneficial or possibly harmful for subjects with COPD. Further studies are also needed to understand the etiology of this response.

The study has some limitations. First, the study population was small and lacked a control group. Furthermore, the study population consisted of a heterogeneous group of subjects with COPD with variations in lung function, medications, spleen volume, $\mathrm{Hb}$, and $\mathrm{O}_{2}$ saturation. Due to the small sample size, only bivariate associations were analyzed. The present studies need confirmation by larger studies with control subjects. The present study population tended to have polycythemia, which is traditionally associated with hypoxia in COPD [34]. It should therefore be investigated in larger studies whether baseline $\mathrm{Hb}$ is an independent predictor of the spleen response.

\section{Conclusions}

The present study shows that exercise evokes acute, transient spleen contraction and $\mathrm{Hb}$ increase in subjects with moderate to very severe COPD and that spleen volume is correlated to the arterial oxygenation. These responses are similar to observations in healthy subjects during maximal exercise or voluntary apnea and may serve as a protection against acute and aggravated hypoxia $[5,18]$. The increased spleen volume in COPD subjects with severe hypoxia at rest could be due to longterm adaptation to hypoxia. The stronger response in subjects with baselien $\mathrm{SpO} 2>90 \%$ may suggest an 
upgraded protective response against hypoxia, similar to the enhanced response found in apnea divers.

\section{Abbreviation}

$\mathrm{Hb}$ : hemoglobin concentration; COPD: chronic obstructive pulmonary disease; SpO2: peripheral oxygen saturation; 6MWT: 6 minute walking test; FEV1: forced expiratory volume first second (liters); FVC: forced expiratory volume (liters); min: minutes; NS: non significant.

\section{Competing interests}

None of the authors have any competing interests to declare in relation to this work.

\section{Authors' contributions}

ES and NS designed the research. All authors contributed in data collection. $\mathrm{NS}, \mathrm{AH}, \mathrm{ALS}$ and $\mathrm{HE}$ analysed the data. All authors contributed in writing of the manuscript. All authors contributed to revision of the final manuscript and approved it for submission. All authors read and approved the final manuscript.

\section{Authors information}

ES and NS are senior authors. NS is senior consultant and ES is professor

\section{Acknowledgements}

We thank the study subjects for their kind participation in this study, and the nurses at the Respiratory Department, Östersund Hospital, for their assistance with measurements. The study was supported by the Swedish National Center for Research in Sports (CIF) and Unit of Research, Education and Development, Östersund Hospital, Region Jämtland Härjedalen.

\section{Author details}

'Department of Health Sciences, Mid Sweden University, Östersund, Sweden. ${ }^{2}$ Swedish Winter Sports Research Centre, Mid Sweden University, Östersund, Sweden. ${ }^{3} \mathrm{LHL}-K l i n i k k e n e$ Røros, Røros, Norway. ${ }^{4}$ Department of Public Health and Clinical Medicine, Unit of Research, Education and Development Östersund, Umeå University, Umeå, Sweden.

\section{Received: 23 August 2015 Accepted: 8 December 2015}

\section{Published online: 29 December 2015}

\section{References}

1. Stewart IB, Warburton DE, Hodges AN, Lyster DM, McKenzie DC. Cardiovascular and splenic responses to exercise in humans. J Appl Physiol. 2003;94(4):1619-26.

2. Qvist J, Hill RD, Schneider RC, Falke K, Liggins GC, Guppy M, et al. Hemoglobin concentrations and blood gas tensions of free-diving Weddell seals. J Appl Physiol. 1986:61(4):1560-9.

3. Hurford WE, Hochachka PW, Schneider RC, Guyton GP, Stanek KS, Zapol DG, et al. Splenic contraction, catecholamine release, and blood volume redistribution during diving in the Weddell seal. J Appl Physiol. 1996;80(1): 298-306.

4. Hurford WE, Hong SK, Park YS, Ahn DW, Shiraki K, Mohri M, et al. Splenic contraction during breath-hold diving in the Korean ama. J Appl Physiol. 1990;69(3):932-6.

5. Schagatay E, Andersson JP, Hallen M, Palsson B. Selected contribution: role of spleen emptying in prolonging apneas in humans. J Appl Physiol. 2001; 90(4):1623-9. discussion 1606.

6. Espersen K, Frandsen H, Lorentzen T, Kanstrup IL, Christensen NJ. The human spleen as an erythrocyte reservoir in diving-related interventions. J Appl Physiol. 2002:92(5):2071-9.

7. Schagatay E, Haughey H, Reimers J. Speed of spleen volume changes evoked by serial apneas. Eur J Appl Physiol. 2005;93(4):447-52.

8. Oka T, Itoi T, Hamaoka K. Impaired transient elevation of blood hemoglobin in response to acute hypoxia in neonates with asplenia. Pediatr Int. 2007; 49(6):898-902

9. Bakovic D, Valic Z, Eterovic D, Vukovic I, Obad A, Marinovic-Terzic I, et al. Spleen volume and blood flow response to repeated breath-hold apneas. J Appl Physiol. 2003;95(4):1460-6.

10. Sandler MP, Kronenberg MW, Forman MB, Wolfe OH, Clanton JA, Partain CL. Dynamic fluctuations in blood and spleen radioactivity: splenic contraction and relation to clinical radionuclide volume calculations. J Am Coll Cardiol. 1984;3(5):1205-11.

11. Flamm SD, Taki J, Moore R, Lewis SF, Keech F, Maltais F, et al. Redistribution of regional and organ blood volume and effect on cardiac function in relation to upright exercise intensity in healthy human subjects. Circulation. 1990;81(5):1550-9.

12. Laub M, Hvid-Jacobsen K, Hovind P, Kanstrup IL, Christensen NJ, Nielsen SL. Spleen emptying and venous hematocrit in humans during exercise. J Appl Physiol. 1993;74(3):1024-6.

13. Richardson MX, Lodin A, Reimers J, Schagatay E. Short-term effects of normobaric hypoxia on the human spleen. Eur J Appl Physiol. 2008;104(2):395-9.

14. Lodin-Sundstrom A, Schagatay E. Spleen contraction during 20 min normobaric hypoxia and 2 min apnea in humans. Aviat Space Environ Med. 2010;81(6):545-9.

15. Richardson MX, Engan HK, Lodin-Sundstrom A, Schagatay E. Effect of hypercapnia on spleen-related haemoglobin increase during apnea. Diving Hyperb Med. 2012;42(1):4-9.

16. Guntheroth WG. In vivo measurement of dimensions of veins with implications regarding control of venous return. IEEE Trans Biomed Eng. 1969;16(4):247-53.

17. Turner AW, Hodgetts VE. The dynamic red cell storage function of the spleen in sheep. I. Relationship to fluctuations of jugular haematocrit. Aust J Exp Biol Med Sci. 1959:37:399-420.

18. Schagatay E, Richardson MX, Lodin-Sundstrom A. Size matters: spleen and lung volumes predict performance in human apneic divers. Front Physiol. 2012:3:173.

19. MacDonald IC, Schmidt EE, Groom AC. The high splenic hematocrit: a rheological consequence of red cell flow through the reticular meshwork. Microvasc Res. 1991;42(1):60-76.

20. Rabe KF, Hurd S, Anzueto A, Barnes PJ, Buist SA, Calverley P, et al. Global strategy for the diagnosis, management, and prevention of chronic obstructive pulmonary disease: GOLD executive summary. Am J Respir Crit Care Med. 2007:176(6):532-55.

21. Antonucci $R$, Berton $E$, Huertas $A$, Laveneziana P, Palange P. Exercise physiology in COPD. Monaldi Arch Chest Dis. 2003:59(2):134-9.

22. ATS. ATS statement: guidelines for the six-minute walk test. Am J Respir Crit Care Med. 2002;166(1):111-7.

23. Allsop P, Peters AM, Arnot RN, Stuttle AW, Deenmamode M, Gwilliam ME, et al. Intrasplenic blood cell kinetics in man before and after brief maximal exercise. Clin Sci (Lond). 1992;83(1):47-54.

24. Engan HK, Lodin-Sundstrom A, Schagatay F, Schagatay E. The effect of climbing Mount Everest on spleen contraction and increase in hemoglobin concentration during breath holding and exercise. High Alt Med Biol. 2014;15(1):52-7.

25. Prassopoulos P, Daskalogiannaki M, Raissaki M, Hatjidakis A, Gourtsoyiannis $N$. Determination of normal splenic volume on computed tomography in relation to age, gender and body habitus. Eur Radiol. 1997;7(2):246-8.

26. Schagatay E, Richardson MX, Lodin-Sundstrom A. Size matters: spleen and lung volumes predict performance in human apneic divers. Front Physiol. 2012. doi:10.3389/fphys.2012.00173

27. Richardson MX, de Bruijn R, Schagatay E. Hypoxia augments apnea-induced increase in hemoglobin concentration and hematocrit. Eur J Appl Physiol. 2009;105(1):63-8.

28. Freedman BJ, Penington DG. Erythrocytosis in Emphysema. Br J Haematol. 1963:9:425-30.

29. Nakamura A, Kasamatsu N, Hashizume I, Shirai T, Hanzawa S, Momiki S, et al. Effects of hemoglobin on pulmonary arterial pressure and pulmonary vascular resistance in patients with chronic emphysema. Respiration. 2000; 67(5):502-6

30. Engan H. Schagatay E. Spleen Contraction and Hemoconcentration Regarding the Review "Hemoconcentration and Hemostasis During Acute Stress: Interacting and Independent Effects" by Austin et al. 2011. Ann Behav Med. 2015;49(4):634-5

31. Austin AW, Patterson SM, von Kanel R. Stress-hemoconcentration: plasma volume changes or splenic contraction? A Reply to Engan and Schagatay. Ann Behav Med. 2015;49(4):636-7. doi:10.1007/s12160-015-9708-1.

32. Chaouat A, Naeije R, Weitzenblum E. Pulmonary hypertension in COPD. Eur Respir J. 2008;32(5):1371-85.

33. Tsantes AE, Papadhimitriou SI, Tassiopoulos ST, Bonovas S, Paterakis G, Meletis I, et al. Red cell macrocytosis in hypoxemic patients with chronic obstructive pulmonary disease. Respir Med. 2004:98(11):1117-23.

34. Cote C, Zilberberg MD, Mody SH, Dordelly L, Celli B. Haemoglobin level and its clinical impact in a cohort of patients with COPD. Eur Respir J. 2007: 29(5):923-9. 